\title{
Collaboration Surrounding Beacon Use During Companion Avalanche Rescue
}

\author{
Audrey Desjardins ${ }^{1}$, Carman Neustaedter ${ }^{1}$, Saul Greenberg ${ }^{2}$, and Ron Wakkary ${ }^{1}$ \\ ${ }^{1}$ School of Interactive Arts + Technology, Simon Fraser University, Surrey, British Columbia, Canada \\ ${ }^{2}$ Department of Computer Science, University of Calgary, Calgary, Alberta, Canada \\ \{adesjard, carman_neustaedter, rwakkary\}@sfu.ca, saul.greenberg@ucalgary.ca
}

\begin{abstract}
When facing an avalanche, backcountry skiers need to work effectively both individually and as a group to rescue buried victims. If they don't, death is likely. One of the tools used by each person is a digital beacon that transmits an electromagnetic signal. If buried, others use their beacons to locate victims by searching for their signals, and then dig them out. This study focuses on the collaborative practices of avalanche rescue and the interactions with beacons while backcountry skiing. We conducted interviews with backcountry recreationists and experts, and we observed avalanche rescue practice scenarios. Our results highlight aspects and challenges of mental representation, trust, distributed cognition, and practice. Implications include three considerations for the redesign of beacons: simplicity, visibility and practice.
\end{abstract}

\section{Author Keywords}

Avalanche, backcountry, snowboard, transceiver, beacon, ski, collaboration, communication, distributed cognition.

\section{ACM Classification Keywords}

H.5.3 [Group and Organization Interfaces]: Computersupported cooperative work; H.1.2 [User/Machine Systems]: Human factors.

\section{INTRODUCTION}

Backcountry skiing is becoming more popular every year with an increasing number of skiers and snowboarders seeking untouched snow, new lines, and challenging but rewarding adventures [17]. When traveling in the backcountry - areas where there is no avalanche control skiers and snowboarders assume the risk of being caught in an avalanche that can lead to injuries or fatalities. An avalanche is loose snow that rapidly slides down a slope [16]. If buried in the avalanche, victims rely on rescue teams for their survival - usually their backcountry skiing companions through a process called companion rescue.

Rescue is far from easy. Avalanche accidents are extreme, stressful and challenging situations demanding efficiency, speed and team effort. Time is of the essence, as the chance

Permission to make digital or hard copies of all or part of this work for personal or classroom use is granted without fee provided that copies are not made or distributed for profit or commercial advantage and that copies bear this notice and the full citation on the first page. Copyrights for components of this work owned by others than ACM must be honored. Abstracting with credit is permitted. To copy otherwise, or republish, to post on servers or to redistribute to lists, requires prior specific permission and/or a fee. Request permissions from permissions@acm.org.

CSCW'14, February 15-19, 2014, Baltimore, Maryland, USA.

Copyright (C) 2014 ACM 978-1-4503-2540-0/14/02 ...\$15.00.

http://dx.doi.org/10.1145/2531602.2531684. of survival decreases significantly after 15 minutes in a complete burial [8]. Collaboration and coordination is vital: the group - typically comprising friends, acquaintances and even strangers of various skill levels - must quickly choose a leader to direct and distribute roles amongst them, and each person must perform their part of the rescue in concert with others if they are to successfully rescue the victim.

As victims can be completely buried under avalanche debris, specialized equipment - almost always carried by the prepared backcountry recreationist - is required to search for and dig out the victim to achieve any chance of survival. Beacons are electronic transmitters/receivers that rescuers use to search for the victim, where rescuers try to identify the snowpack surface directly above or nearby the victim [5]. Collapsible probes (long poles) are used to pinpoint that search, where a rescuer probes that area at intervals to physically strike - and thus find - the victim. Avalanche shovels are used to dig out the victim. See [3, p.27-33] for an account of a real accident.

While there is much research concerning avalanche causes, medical implications, and technological advancement in beacon technology (e.g., $[1,6,8,17]$ ), little research investigates avalanche rescues from computer-supported cooperative work $(\mathrm{CSCW})$ or interaction design perspectives. Currently, much of the focus in equipment design is oriented towards a single user, in particular his use of the beacon. The problem is that the overall success of the rescue relies heavily on the ability of the group to collaborate. Thus the specific goal of our research is to investigate collaboration in avalanche rescue as facilitated by the use of beacons.

In this paper, we explore how groups train with beacons, how beacons fit into daily routines, and how groups use beacons during rescues. We are particularly interested in how existing beacon designs serve well or poorly as a collaborative artifact. Our results show the complexity of this distributed effort and provide insights on the challenges of creating and sharing mental representations of the rescue scene and the quality of trust within the team and with the beacon. We also reveal the importance of the scene itself and how we see distributed aspects of cognition through the acts of the rescuers, the tools they use and the environment they act in. Finally, we discuss the challenges of performing meaningful practice and training. These results lead us to a discussion of visibility, simplicity and practice that can 
influence and re-orient the design of tools for avalanche rescue.

BACKGROUND INFORMATION Backcountry skiing and avalanche rescue are activities that need training and basic knowledge to perform successfully. In this section, we briefly present background information regarding accepted backcountry skiing protocols, avalanche rescue coordination, and avalanche survival technologies. Additional detail can be found in books such as [5].

\section{Backcountry skiing protocol}

Backcountry skiing involving avalanche accidents, while relatively rare, have life and death consequences. Thus, route planning includes analyzing the avalanche and weather forecast, and using that information to select an area within the group's risk tolerance. Equipment is prepared accordingly; typically including the rescue tools mentioned earlier, food and water, a first aid kit, compass and map, and clothing.

On the day of the trip, mountaineers put on their beacon as they are putting on their outdoor clothing. At the trailhead, groups do a beacon check in order to make sure that everyone's beacon is working, i.e. it is emitting a signal and that it can be picked up at a certain distance. To perform the check, one person is elected to be the "searcher" and puts their beacon in search mode. Each person from the group skis by this person, who verifies that he can pick up their signal. Once everyone has passed, the searcher switches his beacon to transmit and (ideally) someone else checks his signal.

Route-finding through the terrain is heavily influenced by the perceived risk of avalanches on particular terrain features. When one has to ski through an area with avalanche risk (e.g., when descending a steep ski slope), the protocol is to send one skier at a time with others acting as spotters. This reduces the risk of the entire group being buried, while allowing other skiers to be well positioned to rescue a skier caught in an avalanche.

\section{Companion rescue}

Almost all avalanche rescues rely on companion rescue, where rescuers are the victim's companions ${ }^{1}$. Companion

\footnotetext{
${ }^{1}$ Professionally organized rescues are normally reserved to areas where ski patrollers and professionals can arrive on-scene very quickly, such as within or immediately around ski areas, or by helicopter-assisted skiing. In recreational backcountry skiing, delays mean that organized rescues are typically targeted towards body recovery.
}

rescues are framed around a prescribed protocol of action, which implies that every recreationist is prepared to learn and practice that protocol. This is why professional bodies strongly advocate that skiers take avalanche courses (although these range considerably in their length and depth of treatment). Prepared skiers will train with their equipment several times a year, either individually (e.g., practicing beacon searches) or as a group (e.g., running through avalanche scenarios). In reality, practice and experience levels within and between groups vary considerably.

\section{Leadership and collaboration in avalanche rescue}

Collaboration is an important aspect of companion rescue. The first step taught in classes and emphasized in training sessions is to choose a leader who will overview the scene and assign roles to other rescuers (e.g., searchers, shovelers, probers, lookouts). The leader assigns each searcher a search path to ensure that: (a) every area of the avalanche scene is being searched and (b) no victim is left buried on higher terrain.

\section{Rescue protocol for burials}

Once the leader has assigned roles, the next step is the coarse search. Those chosen as searchers start at the victim's last seen point (Fig. 1). They move downhill searching for physical signs of the victim (e.g., a body part above the snow) and for a signal with their beacon. Because beacons have limited range, searchers must coordinate their search into a pattern. The idea is that their collective patterns of search will cover the entire avalanche path. For example, a single rescuer on a wide path may follow a wide $\mathrm{S}$ pattern to ensure the whole avalanche area is covered. As more people search, each person's S-pattern can be narrower (Fig. 1), until all are descending in a straight line.

The beacon signals when it first acquires another beacon's signal (which should be the victim's beacon) and provides cues to guide the searcher towards it (described in the next section). When the searcher is close to the victim, she starts a fine search to find the closest point on the surface to the victim. The searcher moves the beacon across the snow surface to discover the area covered by the beacon's highest reading, and marks those as boundaries. This area may range from .2 to $2 \mathrm{~m}$ or more in width, depending on the depth of the burial. Within this bounded area, the rescuer(s) then systematically probe into the snow pack until they 'touch' the victim, after which they start shoveling to create an airway to the victim. During this process, rescuers shout about their activity and needs, e.g. "I got a signal" or "I need a prober" or "I need some shovelers". 
With multiple burials, the leader must monitor the scene to ensure that searchers do not congregate on a single victim at the expense of others that are still buried. Even with this guidance, communication amongst teammates is the weakest link in many rescues and it is often what makes the difference between survival and fatality [6].

\section{The Beacon}

Various tools are available to backcountry skiers to increase their chance of survival in the case of an avalanche [2]. We focus on the beacon, because it is the most common tool used by backcountry recreationists [5], and because it is the only tool that realistically shortens burial duration.

While beacons vary considerably, all have two primary modes of operation, illustrated by the sample beacon in Figure 2. In transmit mode, it broadcasts an electromagnetic signal of $457 \mathrm{kHz}$ along a radial flux line pattern ${ }^{2}$ (Fig. 1, dashed black lines): a blinking light provides feedback that it is active (Fig. 2a). In receive mode, it displays auditory and visual information to help a person search for this signal [9]: a digital numeric display estimates the buried victim's distance (here $\sim 35$ meters), the approximate direction to the victim (the small arrows), and audio feedback as an intermittent beep that increases in speed, pitch and/or volume as the rescuer approaches the victim's beacon (Fig. 2b). Distances and direction are approximate, as they follow the curved flux lines of the signal (Fig. 1) rather than a linear path. If multiple burials occur, the light below the number turns on to signal multiple victims and the beacon will display alternating signals for each victim. Some beacons allow the searcher to mark (hide) certain signals, allowing one to concentrate on other signals. ${ }^{3}$

\section{RELATED WORK}

While seemingly very specific, avalanche rescue relates to CSCW theories relevant to emergency and high stress situations. There is also a smattering of HCI work on the human factors of avalanche rescue beacons.

\section{CSCW theories relevant to stressful and emergency situations}

Within CSCW, various theories of social behaviors have focused on small teams of people who must coordinate their

\footnotetext{
${ }^{2}$ Beacon signals emanate in space as a flux field: a curved 3D aura centered around the beacon. Figure 1 illustrates a cross section of this 3D field as curved flux lines. Thus, a searcher following a signal's flux line first moves in a slight curve away from the victim, and then back towards him. 'Dead spots' are also present along the antenna axis, affecting fine-search accuracy. Modern transceivers mitigate this through multiple antennas.

${ }^{3}$ Beacons vary considerably in how they track, manage and display multiple signals. Algorithms distinguishing such signals are quite complex where, for example, rapid movements can confuse this tracking.
}

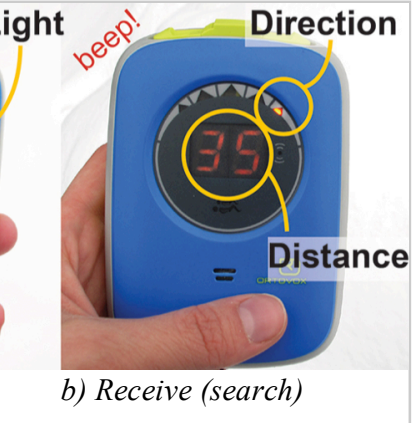

activities. These theories contribute to our ability to define, structure, analyze and interpret studies of collaborative teams and the development of interactive tools in stressful and emergency situations.

Hutchins' framework of distributed cognition [10,11] is most relevant to our study of collaborative avalanche rescue [3, p.27-33]. Distributed cognition argues that in many situations 'cognition' involves social aspects: the coordination between individuals (e.g., their social interactions and their social relationships), and how people coordinate their interactions between themselves and the artifacts within their collective environment. Thus distributed cognition is fundamentally embodied, where the body and the world are central to cognition aspects. It is also part of a cultural context where people accumulate partial solutions to situations over time and are able to use these in new situations [10]. Hutchins originally used distributed cognition to study ship navigation and to highlight the importance of the interactions between multiple navigators and their various tools on the ship [11]. We believe distributed cognition is highly relevant to the study of emergency situations like companion rescue: overall coordination of the situation relies on how individuals monitor and interact with other team members, the use of the beacon as an artifact, and how all are situated in the surrounding environment.

Implicit collaboration is a construct within distributed cognition that "describes the ability of team members to act in concert without the need for overt communication" [13, p.2]. Implicit collaboration is necessarily a part of efficient emergency teamwork (including companion rescue) since it allows teammates to concentrate on tasks to achieve and reduce the amount of explicit communication they have to do. For example, studies of firefighters highlight the importance of tacit communication [12] and implicit coordination [19]. Toups and Kerne [19] state that clear shared mental models are crucial to good collaboration in stressful and extreme situations because they eliminate the need for explicit communication and allow the process to be "streamlined" [p.714].

Situation awareness (SA) is "the perception of the elements in the environment within a volume of time and space, the comprehension of their meaning, and the projection of their status in the near future" [7, p.36]. SA highlights the importance of how a person understands 'what is going on' in order to help the decision-making process in dynamic domains such as aircraft piloting [7], ship navigation [11], air traffic control, and tactical situations like those involving firefighters [19], police, or military command personnel. The SA theoretical framework includes three 
levels: perception, comprehension, and projection. In brief, SA is necessary in emergency situations like companion rescue: it allows people to understand the situation, the context they are in, to predict what might happen next, and to take decisions accordingly as they conduct an efficient rescue.

The context of avalanche rescue is somewhat similar to how others have investigated stressful and emergency situations. Companion rescue, like some other dynamic domains mentioned above, require potentially life or death decisions. As we will see, distributed cognition, situational awareness and implicit coordination manifest themselves in successful avalanche rescues, similarly to how firefighters, pilots, and ship crews rely on the same constructs. Companion rescue, however, has its own unique circumstances. Recreationists - who may not have worked together in a rescue situation before - become ad hoc rescuers. Individuals are mostly non-experts, and thus their familiarity with technology can considerably influence the team effort. The situation is highly unpredictable due to weather and terrain features, the location of people (who, for example, may be scattered around the slope), the abilities of the rescuers, the risk of further avalanches, fatigue, cold, and so on.

The professional avalanche community is not blind to the collaborative nature of companion rescue, although they rarely use the above theoretical constructs to frame their work. The new generation of both research $[1,14]$ and avalanche safety classes [18] focus on group dynamics and decision making surrounding backcountry skiing, such as "tools for planning, communication and group management" $[1,6]$. Classes teach students to be familiar with beacon use through simple exercises such as finding a buried beacon under snow (an individual activity to gain device proficiency), to more elaborate settings such as avalanche scenarios with multiple burials (a group activity).

\section{Beacons and $\mathrm{HCl}$ research}

Beacon design has evolved over the years, in part by advances made by manufacturers and in part by feedback from avalanche rescue professionals. However, most have centered on increasing an individual's performance rather than considering the beacon as a collaborative artifact. For example, Hereford and Edgerly propose that future avalanche beacons include an additional signal that uniquely IDs each mountaineer [9]. This would help rescuers separate multiple signals in multiple burial situations, particularly in scenarios where two victims are situated very close together.

One noteworthy exception is the work by Michahelles et al. [15]. At issue is how significant time may be lost uncovering dead victims or victims who may be deeply buried at the expense of other live victims. Their focus was to redesign beacons to support triage, where the team decides which victim to rescue first, and thus where their efforts should be concentrated. They added sensors that collected and transmitted data about the victim to support triage: heart rate, oxygen blood level, victim orientation under the snow, and depth of the burial.

The aforementioned theories as used in CSCW help us frame our study of avalanche rescues in order to focus on the collaborative aspects of the rescue as well as the interactions with the beacons themselves. By using this perspective, we contribute research on beacon technological advancement and human factors in avalanche rescues.

\section{METHODOLOGY}

In order to gain an overview of the collaborative rescue practices and the use of beacons, we used two methods for collecting data: semi-structured interviews with experienced backcountry recreationists, and observation of practice avalanche rescue scenarios. We chose these two qualitative inquiry methods because of our study's explorative nature, where they would help (a) uncover the broad and holistic collaborative practice of avalanche rescue and the use of beacons [4], (b) triangulate findings, and (c) provide more diverse and in-depth exploration than a single method [20].

\section{Interviews with recreationists and experts}

We met with ten participants (one female), where individual backcountry expertise ranged from 2 to 30 years of experience. Four were recreationist backcountry skiers. The other six were considered experts in the field of avalanche control or education: one ski patroller, one backcountry skiing guide, three avalanche safety instructors and one researcher in avalanche snow and weather conditions in Washington, USA, and in Alberta and British Columbia, Canada. Participants were chosen through snowball sampling. They were not remunerated.

For each participant, we conducted a semi-structured interview in person, or via phone or Skype: interviews lasted from 30 to 90 minutes. Hand written notes were collected and used to create a report for each interview. Interviews included questions related to three topics: backcountry traveling history, avalanche rescue situations, and the use of beacons and tools in avalanche rescues. We started with ice-breaking questions to learn about the participant, his goals, and his experience and motivations for doing backcountry skiing. We then focused on the two aspects that are closely related to $\mathrm{CSCW}$ and interaction design and that are missing from the literature: the aspects of collaboration and the use of tools (i.e. beacons) by the participants. Lastly, we opened the conversation to discuss challenges and potential improvements in the future development of beacons.

\section{Observations of avalanche rescue scenario}

In order to gain more insight on how beacons are really used in avalanche settings, one of the authors observed avalanche scenario sessions at the Mount Baker Ski Area in Washington, USA (another author is also a domain expert). Since it is very problematic to observe real avalanche 
rescues, these scenarios were the next best opportunity to see how people use beacons in a time-constrained and real environmental setting. We observed two types of groups doing avalanche rescue scenarios: three groups of five students of a MEC 2 class (with varied levels of experience typical of companion rescue teams) and two groups of seven patrollers from the Mount Baker Ski Area (with high level of experience). The MEC 2 class allowed each group of five to practice three different rescue scenarios on the mountain (total observation time: 3 hours). The pro patroller groups performed one rescue each on the mountain (total observation time: 5 hours). In both cases, we collected hand written data about the team organization and process, and how the beacons were used. In the both patroller groups, we videotaped the scenarios. Since things happen quickly in these situations, video proved valuable for documenting otherwise hard to observe information.

\section{Analysis method}

All data was analyzed with a thematic analysis. We extracted themes that could be clustered in three areas:

- team collaboration during an avalanche scene (including search steps, multiple burials situations, general protocol on scene, and the beacon in the environment),

- the beacon itself (including physical aspects, technical aspects, use issues, potential additional functions, and the simplicity of the tool), and

- training and practice aspects of using beacons (including education, practice, skills and beacon checks).

We now present the results and analysis based on our data. Data from interview participants is labeled with P\# for participant number, while observational data is labeled with O\# for observational participant.

\section{CREATING MENTAL REPRESENTATIONS}

On the avalanche scene, rescuers work to construct a representation of where the victims might be buried based on the information provided by the beacon and by other physical hints in the environment. To work as a team, rescuers must try to share this mental idea with others. However our data show the various challenges in doing so. Some of these come from challenges in how people share information, while others come from an individual's difficulty in obtaining and interpreting beacon readings.

\section{Sharing mental representations}

In scenarios with multiple burials and multiple rescuers, rescuers collaborated to find the buried victims by partitioning the search zone. That is, one person may search one area while another person may search an area lower down the hill. The goal is to not repeat search areas. This can be challenging since the second searcher does not have the same mental representation as the first searcher who was able to pick up the signal from further up the hill.

"It is hard to pick up on someone else's signal. The person who found the first victim has already an idea of how the signals are present in the scene and should be the one

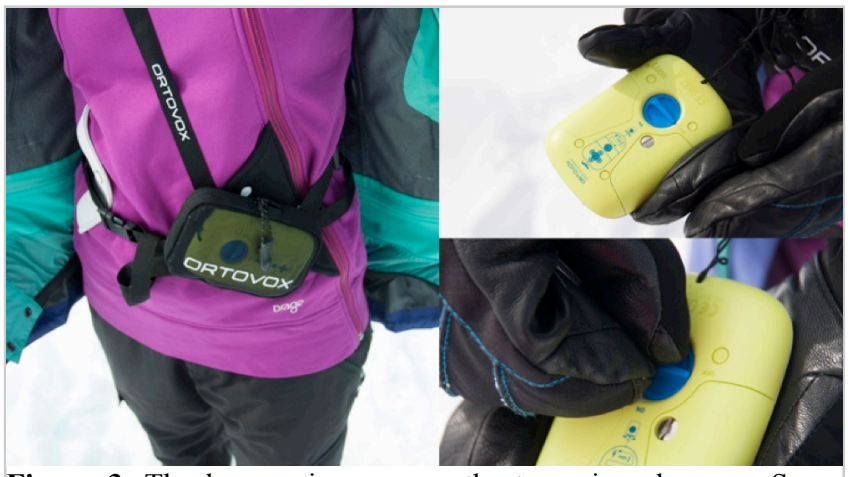

Figure 3. The beacon is worn on the torso in a harness. Some functions and buttons are challenging to use with gloves.

continuing to the second victim. He already has this mental model. More intuitiveness for where to go." - P4

Thus shared mental representations between rescuers in the group are extremely important, especially in the case of multiple burials. Yet we observed that the information gathered from one beacon by one rescuer is not often communicated to others on scene. Instead, each individual rescuer is working hard to understand his beacon and create one's own accurate mental representation (see below). There is little time to discuss another person's mental representation, let alone understand it.

\section{Single burial challenges}

Even before sharing a mental representation with others, rescuers need to first create a representation for themselves of the possible location of victims. We observed multiple challenges in the design of beacons that constrain the collaborative process. First, P2 and P10, two instructors, said that many backcountry skiers do not understand that the beacon functions by tracking the non-linear radial 3D electromagnetic signals (Fig. 1, dashed black flux lines). Because the signal follows the curved flux lines, the orientation will change as the rescuer moves on the site. This means that distance and orientation readings are at best approximations; while knowledgeable searchers can make assumptions and conclusions based on how this information changes, this is hard for those with fewer skills. In order to assess potential places for burial and create a mental image of where the victim might be, rescuers need to understand and apply this knowledge of the beacon's operation. Another problem is that rapid movements of the beacon, including beacon rotation, will affect how the beacon discriminates between signals and how it estimates direction and distance. Thus, success hinges in part on a rescuer's ability to correctly hold and move the beacon, and to correctly understand, interpret and make decisions based on its information in an efficient manner while under stress.

The second problem in creating an accurate mental representation arises from issues with device ergonomics. One example is difficulties in correctly reading beacon information. We observed participants of the MEC 2 class having difficulty reading the beacons' distance numbers. 
For example, one participant was not able to differentiate " 2.0 " from " 20 " or "1.0" from "10". Similarly, P10 mentioned that older mountaineers can have difficulty reading the display without reading glasses, which are rarely handy. Obviously, such misreads could dramatically slow the search. Another example is that most beacons are physically difficult or awkward to handle (Fig. 3). When worn under the clothes, beacons must be extracted. Because they are usually tethered to the body in an elastic harness, they are sometimes difficult to move to particular positions. Because people wear gloves, their controls are at times difficult to use.

\section{Multiple burial challenges}

All experts in our interviews told us that multiple burial accidents were the most challenging rescues to perform. P4, P7 and P9 said that separating two signals was the hardest thing to do with a beacon, e.g., "it needs practice since it is not intuitive" (P7). To explain: as the searcher moves, the beacon indicates smaller or bigger numbers on the display. While modern beacons try to 'lock onto' the strongest signal, the process is imperfect. Thus they may flip between the data readings for each buried victim (Fig. 4). This often results in what appears to be contradictory or random readings, or signals that change unpredictably. P2, P4 and P9 further explain that in order to perform a multiple burial search successfully, the rescuer needs to use the readings on the beacon to track the beacons worn by all victims within range. This is difficult: the rescuer must retain several mental images or construct a more complex representation. For many first-time users, this was the most challenging aspect of understanding the beacon's readings. We observed in the MEC 2 class that most participants were not certain if they were still picking up the first signal they found or if they were on the right path to look for a second victim. For example, one participant said: "I keep getting a soft second reading but I can't pinpoint it" (O1).

Most advanced digital beacons remedy this somewhat by allowing the searcher to 'mark' a signal during multiple burials, which removes it from the display. However, we did not observe the MEC 2 participants using this function on their beacons: they indicated that they simply did not know how to use it. Even so, marking has its own associated issues that require expert interpretation, e.g., marked signals may 'come alive' due to the difficulty the device has in differentiating between competing signals.

\section{BUILDING TRUST}

Sharing information (e.g. mental representations) and coordinating tasks on scene are crucial for the team to perform successful rescues. These activities, however, also rely heavily on social dynamics. A theme that appeared recurrently in our data analysis is trust: trust in the other rescuers on site, and trust in the beacon.

\section{Trust between team members}

Our interviews and observations revealed that team members must work together in both tightly-coupled and loosely-coupled situations, where trust is vital to both.

\section{Loosely-Coupled Collaboration}

Loosely-coupled collaboration occurs when rescuers work together but each has one's own specific task and goal. As mentioned, each rescue group appoints a leader and the leader assigns different search roles to people in the group. This is challenging in companion rescue due to the lack of information about team member abilities, especially in situations when skiing groups are created ad hoc at the trailhead or in the ski area. For example, when choosing who will be doing the beacon search, a leader often does not know who has the best search skills. Trust in the leader's decisions as well as the abilities of the other group members to perform their assigned roles within the group may be easily questioned.

When groups know each other very well, the distribution of the roles and the collaboration between rescuers can be fluid and efficient. We observed a close collaboration effort in the scenarios with the pro patrollers who worked together as a team on a daily or weekly basis.

"We were all really fluid in our roles. We all know each other and can switch between roles. But this would not work with a different group and customers." - O2

We observed that clear communication between rescuers allowed for faster searches. Rescuers that were not beacon searchers were aware of the scene and ready to come help with the tools for the next steps. We observed implicit coordination in the pro patrollers teams where roles were established and understood without explicit communication.

In contrast, in the MEC 2 class, groups comprised people that did not know each other; hence, the teammates did not find a leader as fluidly as the pro patrollers. However, since each group performed three rescues, we observed a clear evolution in the leadership roles and team sense from the first rescue to the last rescue. This led to improved collaboration and trust, which ultimately can help achieve more efficient rescues.

\section{Tightly-Coupled Collaboration}

Rescuers also work in tightly-coupled situations, where two or three rescuers typically work together to rescue the same 
victim. For example, as one person fine-tunes the victim's location, another may start probing or shoveling. In these cases, precise coordination is necessary to know when the beacon search is advanced enough so that the other tasks can efficiently be started. Hence, team members must trust the efforts of each other and also relay information while searching. Team members must trust that the information shared with them by other team members is accurate.

To illustrate, one rescuer in the pro patrollers group did a fine search with the beacon and set the area for the second rescuer to probe the snow. The second rescuer started to probe and did not get a strike even after probing multiple times. The instructor asked him to redo the beacon fine search, however, the rescuer trusted his partner and decided to continue to probe. A few seconds later, he probed and found the victim. This is in an interesting example since it shows the trust and confidence a rescuer has in his teammate's abilities. We also saw the sharing of mental representations about the scene: the rescuers shared the information they had through signals with their hands by pointing at the area and the center of the fine search results, and the searcher with the beacon used lines he made in the snow to show to the prober where to start probing. In brief, team members trusted each other's abilities to perform their tasks, but also used visual clues to indicate the mental representation they had about the victim's position. In contrast, ad hoc companion rescue groups rarely have the opportunity to form this tight-knit expertise relationship.

\section{Trust in the tool}

In some cases, a lack of understanding of the flux lines and the shape of these lines brought multiple questions, confusion, and frustration to less experienced beacon users.

For example, one participant of the MEC 2 class was walking in circles on the scene and she said three times within a few minutes interval: "I don't trust it. I just don't trust it" (O1). As she was getting various readings, she did not know where to direct herself and how to manage this information. She started to doubt her tool and was not able to find a victim without the help of her teammates. Other participants said "the readings were lying to us", "the beacon is confused", and "the beacon locked on one victim" (O1). In all cases, the beacons were functioning normally and it was the interpretation of the signals that participants were struggling with. In situation awareness terms, the first step of situational awareness - perception - is lacking. P2 argued that these questions and lack of trust come from a misunderstanding of how a beacon follows a flux line. These situations were also used by the instructors to remind participants that although the theory of using a beacon is rather simple, the execution can be more challenging.

Beacon precision was also a challenge for rescuers and limited how fast they could do the fine search. Confusing and unreliable readings can occur if the beacon is moved too fast since it does not have time to interpret the buried beacon signal. In order to counter this, some instructors, like P9, encouraged students to physically place the beacon on the snow, read the signal, then pick up the beacon and move it $20 \mathrm{~cm}$ away and repeat. Here, trust between the rescuer and the beacon is built by consciously creating the best context for the beacon to show reliable data.

\section{Perceived simplicity of beacons}

All participants told us that beacons have improved dramatically in the past few years. Participants with less experience explained that they saw the beacon as a very "straight forward" (P5), "easy to use" (P6), and "not confusing" (P1, P6) tool. These participants perceived beacons as simple and passive technologies: "beacons are a very passive technology, invisible technology. You put it in the morning and then you forget about it" (P5).

Interestingly, the more experienced participants generally presented a different perspective. Through their work and experience, they had seen many cases were beacon use broke down and users were not able to use them properly, particularly when there was a lack of practice and training. Thus, we observed a tension between how participants perceived the ease of use of beacons, how challenging they can be in practice to use and how the lack of trust in the beacon can bring doubts and hinder rescue efficiency.

\section{DISTRIBUTED COGNITION ON THE RESCUE SCENE}

Our data showed that distributed cognition and an understanding of the broader scene and environment are important aspects of avalanche rescues. As presented in the related works section, we know that distributed cognition allows people to share what they understand through the acts they are doing with their artifacts and in the environment. This way of externalizing and embodying cognition should be central to the teamwork happening between avalanche rescuers on an avalanche scene. However, we observed that different aspects of the team strategies and of the beacon itself hinder fluid distributed cognition.

\section{Ephemeral and invisible data}

First, we found that there was no way to record beacon data on what had been searched and what had not. Thus it was impossible to see and visualize this data on the scene. Moreover, as soon as a rescuer moved, the data she had in one position was lost and replaced by new data. During the observations of both the pro patrollers and the students in the MEC 2 class, we saw rescuers asking others (and sometimes the leader) if certain areas were explored yet. For this reason, rescue leaders in both groups often stayed at the top of the avalanche scene in order to keep track of what has been explored and to communicate that to the team. Yet this was also challenging as leaders would need to survey and understand what had been searched from afar. In addition, the leader is in charge of depicting a clear and dynamic picture of what is going on: mostly knowing how many victims have been retrieved, how many are still unfound. The leader must also be aware of the role of each person, the problems they encounter, and reassign them as 
needed. All this is difficult to achieve since rescuers might be far away from the leader. Sharing information between the rescue team becomes challenging: verbal or gestural communication might not be possible due to distance, or because searchers are so engrossed in their local activity as to effectively ignore those communications. All this leads to a breakdown in explicit and implicit communications.

During beacon search in multiple burials scenarios, we observed some rescuers who were closer together than was necessary on the terrain. This was because rescuers do not often evaluate and visualize how far their beacon can search. For this specific reason, during training P7 said he had groups start a beacon check close together but then walk as far away as they can from a transmitting person until that signal could not be received. The group's spread provides a visual image of their beacons' range. However, on scene, rescuers cannot 'see' how far their search tool can pick up a signal and align properly with others on site. Another problem arises when two searchers target the same victim without realizing it and thereby reduce the number of searchers looking for victims buried elsewhere. This can easily arise because search zones overlap (e.g. Fig. 1).

\section{Being aware of others and the environment}

Rescuers also need to be aware of the other rescuers on scene and to understand if everyone in the group has switched their beacons to search mode from transmit. This is the first thing rescuers must do when they are on an avalanche scene so the searchers do not accidentally pick up their signal instead of the victims'. Yet this first step is often missed in scenarios and brings much confusion to the scene: searchers end up 'chasing' the moving signal of another searcher. A similar situation arises if the beacon turns back to transmit by error (e.g., many beacons autorevert to transmit mode after a timeout period). Such errors can be detected by practiced rescuers who detect unusual signal changes as part of their situational awareness, but this comes at the cost of time.

Distributed cognition also states that a part of cognition happens through the environment. Rescuers observe the terrain and use this information to support and orient the interpretation of the beacon's reading. However rescuers tend to rely on the beacon to create a mental representation of where the victim could be despite instructors constant refrain that students should also focus on the site to find additional clues from such features as the terrain shape (which suggests where victims may come to rest after the slide) to pieces of personal equipment on the snow surface (e.g., a ski pole). P7 and P9, both experienced avalanche safety instructors, explain that "your brain is your best tool" (P9) meaning that additional thought is needed to take the information provided by the beacon and mentally map it to real world geography. Yet switching one's attention between the beacon and the environment is challenging, since once a rescuer finds a signal, most of his attention is focused on getting as fast as he can to the victim's location.
We observed that both new users and pro patrollers tried to "beat the myopia of the device" (P8).

\section{PRACTICE AND PREPAREDNESS}

Challenges presented earlier - misunderstanding of how the beacon sends and receives signals, sharing mental representations, identifying searched $v s$. unexplored territory, dividing the avalanche scene between rescuers, and how to trust a beacon - could, in theory, be mitigated by good training and practice. P8, an instructor and head of the mountain safety education department, argued "beacons [are] just outside the intuitive realm that they need practice." However, our interviews revealed that a variety of challenges occur in practice.

\section{Lack of seriousness and attention}

The first challenge is that, naturally, practicing is not the preferred activity. The main goal of recreationists is to go skiing. Hence, any time used to practice as a group can be seen as a waste of precious time that could be used on the mountain to ski. Practice time is also often seen as "lame, dry and boring" (P6). Weather also affects this: practice in cold or wet conditions is far from pleasant. In consequence, there is a chance that skiers will not practice, or will not take practice seriously, or will not be fully focused on the practice and what they need to do. P2, P5, P8 and P9 all mentioned that, from their own observations as instructors or guides, recreationists need to practice more. Some participants talked about trying to make practice time fun. For example, when P6 practices with his group, they try to make it more fun by placing rewards with the hidden beacons or by timing everyone in the group to bring more competition and excitement. While beneficial, this can easily present a mismatch in the goal of a practice scenario and associate rescue attempts with the wrong frame of mind. This opportunity could also be used to build team trust and observe each other's skills in preparation for distributing roles on a real avalanche scene. However, this was rarely reported in our interviews.

\section{Beacon check}

We mentioned that beacon checks should be done by the ski group before entering the backcountry area [16]. However, our interviews revealed that beacon checks are not done systematically and that the group of people and the chosen area of skiing influenced this decision. For example, P2 explained that for shorter runs close to the ski area, it felt less important to complete all the safety steps. He also explained that if he travels with people he knows better, he sometimes skips this step. This finding points to human factor considerations, where $\mathrm{P} 2$ argues that we need to find a way to encourage beacon checks.

Although there are multiple ways of doing a beacon check, in most situations the group concludes the beacon check with one person not being checked. Since one person turns his beacon to search, he can check everyone else; however, when he is done, others may not check him as they have already skied by. 


\section{The generalizability of mock practice scenarios}

Beyond the need to simply practice more, we also found that the way recreationists practice is not always ideal and does not represent the reality of an avalanche rescue. Because of the time required to set up practice scenarios that were similar in nature to a real avalanche, some participants talked about overly simplistic forms of practice. For example, P3 hides a beacon in the parking lot before leaving for a day of backcountry skiing. The beacon is not under snow and is not on a slope. Similarly, P1 reported that he hides a beacon in a backpack and buries it under snow on flat terrain. In this case, the challenges brought by an angled slope cannot be tested and practiced. These unrealistic scenes also mean that one does not learn how to interpret the avalanche area for additional cues of where the victim may lie. Yet due to their 'success' in these simplistic scenarios, recreationists may feel overly comfortable and confident with using beacons in a search.

As we described earlier, avalanche rescues are rare and companion rescuers themselves may not be experienced or up-to-date on their practice. This reveals the challenge of designing tools that can easily be picked up and used correctly in a stressful situation by such rescuers. As we observed in the MEC 2 course, even a practice scenario can bring a high level of stress and a range of emotions to the participants. One of the students said that "I know this is just practice, but I can't stop thinking I could be searching for my husband or for my daughter" (O1). In a real avalanche accident, these emotions are even stronger. Yet there is no way of truly simulating a practice scenario with the extreme emotional conditions that rescuers would face in a real avalanche.

\section{DISCUSSION}

Our results reveal that many challenges in collaborative practices of avalanche rescue and use of beacons come from: a lack of regular practice; a lack of understanding of the beacon itself; or from deficiencies in the human factors of beacon design. In order to help recreationists coordinate better on a scene and understand and use their tools better, we propose three considerations in beacon redesign: visibility, simplicity and practice.

However, these considerations are provided with a large caveat: they are not guidelines, nor should they be even considered correct. Rather, they are best viewed as broad suggestions for those exploring the future design of safety tools in avalanche conditions, especially for designs that depart considerably from the current status quo. We recognize that much work would be required to produce 'good' designs from these implications, not only because of human factors issues but because of limitations in beacon technology. Even if reasonable designs are found, much work needs to be done to see if they are provable better than existing beacons, and if the improvement is worth disrupting current technology standards and avalanche protocol practices.

\section{Visibility}

We found that working with 'invisible' signals was an issue in several situations: a) coordinating how much space to leave between rescuers on the hill to do the coarse search, b) understanding the flux line between the searcher's beacon and the victim's beacon and sharing this with others, and c) differentiating multiple signals in a multiple burial scene. We believe that these three challenges are related to the lack of information rescuers have about the flux line and how it would be represented on the terrain. Moreover, rescuers have trouble sharing or knowing what has been searched on the terrain and what has not. Challenges were also present for rescuers trying to keep track of what signals were being followed and which ones were yet to take care of. In both cases, the lack of visual feedback made it difficult to share or track this useful information.

In order to increase distributed cognition and better teamwork, we believe that the first step is to allow the translation between the distance and direction on the beacon and the physical space to be clear (this could only work if beacon algorithms were able to estimate this information accurately from the data it collects). Only the more experienced backcountry skiers were able to explain how they can construct a representation of the scene that allows them to perform the search faster. This mental image is not only based on the beacon's feedback but is also informed by keeping situational awareness. However, even within their description, they were not able to pinpoint where the victims were, let alone share that with others. Instead, they were only able to approximate the victim's area.

In this case, tools that support the 'seeing' of beacon data within the terrain could be extremely valuable. For example, one interviewee suggested that future beacons could have a larger LCD screen showing the position of the victims in relation to the terrain and landscape features. This would allow users to gain even more information from the beacon and reduce the amount of assumptions they have to make to reach the victim. This strategy, although potentially efficient for orienting oneself on the scene, would require the rescuers to constantly keep their eyes on the device. In addition, creating a way to combine and show information collected by networked beacons held by multiple rescuers could help keep a connection and awareness between rescuers in multiple burial situations. This would also support a structured way of keeping track of the process towards different victims.

There are certainly technical issues as to whether beacons can individually or collectively create an accurate view of the scene based on the limited data they can gather. Even if they could, there is a fine balance between having a tool that shows 'everything on the screen' and a tool that allows the user to take his eyes off the screen to be more aware of the environment. We mentioned that expert users emphasize the importance of maintaining an awareness of 
the physical environment. One possible - albeit quite futuristic - way to mitigate this problem is an augmented reality approach that integrates the virtual view of beacon data with the physical scene.

\section{Simplicity}

Our results also point to the importance of simplicity in the design of beacons used by backcountry recreationists. Questions and confusion can arise because of the lack of simplicity. Practice and training are necessary because beacons are not simple enough to be picked up and used immediately. Since avalanches are rare events, even if recreationists wear a beacon every time they travel in the backcountry, they can use them inefficiently in a stressful and urgent situation like a companion rescue. For this reason, we believe it is useful to design with casual users in mind, and assume that users have only modest training and minimal practice. While our findings revealed a perceived simplicity of the beacon by inexperienced users, experienced users understood the subtlety and complexity of beacon search. This shows that beacons are precise instruments that require practice to lead to proficiency and adding more functionality would only delay this.

Simplicity also relates to proposed additional functionalities for beacons. We previously mentioned that unique IDs can better identify beacons in multiple burials [15] or that sensors could afford display of vital signs [9]. Yet unless great care is taken, this can make the interface and beacon visuals more complex, thus negating possible benefits. With simplicity, we advocate only those functions that keep people directly on task; to the extent that extra information would be helpful, they must be seamlessly integrated into the beacon's visual representation so that the scene can be understood at a glance. Removing less-critical functions implies less decision-making, less unnecessary team coordination, and less analysis of the beacon's interface while on the avalanche scene ${ }^{4}$. Interviewees also argued that all additional functions will require more practice: while potentially useful for guides and patrollers, they would not be appropriate for recreationists. There is clearly a tradeoff here: while beacons should be enhanced to increase their accuracy, interface and representation complexity and the need for interpretation needs to be kept to a minimum.

The unique ID and sensor enhancements proposed by Hereford and Edgerly [9] and Michahelles et al. [15] include tools for a faster triage of victims in a multiple burial setting. While these make sense for professional

\footnotetext{
4 Indeed, some beacons are already designed for simplicity, with the casual user in mind. Without getting into detail, some beacons provide a wide range of settings in how signals are presented on the audio and visual channel as the search progresses, which is useful for those experts who understand signal nuances. Other beacons are designed for casual users there are few or no options, and only a single way to look at the signal. While less confusing for casual use, information is lost for those who know how to exploit it.
}

searchers who may not know the victims, they do raise significant issues for casual teams with differing interpersonal relationships. Both P8 and P10 felt that these additional functions would 'get in the way' and create conflict on the avalanche scene. In our interviews, participants expressed concern around the ethical issues of such a function. "It makes you ask the question - who do I dig out first? I would want to know where my wife is. Would that be fair?" (P5). Indeed, one of the authors of this paper was involved in an avalanche burial with multiple victims (all survived) - an account is found in the opening chapter of [3]. During the search, it was likely no coincidence that the first person to arrive at each victim's site was that person's mate. Thus we argue for safety tools that are not only simple in their interface, but also in the ways they shape the very real human emotions and choices surrounding the search process. Additional triage information can create ethical questions: this can potentially hamper decision-making happening on scene that can cost precious rescue time and lead to bad choices.

\section{Practice}

Finally, we reflect on the importance of practice with safety tools and as a group. One of the interviewees, a ski guide with 30 years of experience, compared beacon search with wood working: "Because I have been making furniture for 40 years, my hands know how to do it. It is automatic" (P9). He argues that beacon search has to become as automatic as practicing a craft at length. In order to help rescuers gain a better understanding of their beacons, we advocate different training tools and environments that would highlight (and maybe make visible) different aspects of the beacon and how it is used over time by a person. These training aids should also consider group aspects and allow recreationists to better structure their practice time to strengthen and reflect on both the tool and the group activities. For example, practice beacon parks - a series of beacons permanently buried under snow that are placed for practice - now allow recreationists to practice beacon searches. We foresee that such parks could track a team's movements, and allow them to 'play back' their search efforts for review and reflection. By practicing together, teams could reach more powerful implicit coordination in the event of an accident. Certain tools could also support the development of routines for critical aspects of the tightly-coupled collaboration, such as when to start to probe while another rescuer is still completing the fine search.

In summary, we believe that avalanche rescue performance can be partially improved by redesigning beacons - if possible - to support distributed cognition. However, this must be supplemented by training tools and practice strategies oriented to increase situational awareness and distributed cognition.

\section{CONCLUSION AND FUTURE WORK}

We contributed a description of avalanche rescue practice and the use of beacons. More generally, our results also 
point to the added need to support distributed cognition in emergency situations managed by non-professionals.

Unlike other emergency situations studied in CSCW, companion rescues are usually performed by non-expert casual users, which reveals unique issues. Our results present multiple challenges in using the beacon that are barriers to accomplishing better teamwork through distributed cognition or situation awareness. Challenges emerge in creating and sharing mental representations of the scene, trusting the rescue team and the tool itself, being aware of what is on scene and what other rescuers are doing, and finally in practice and training contexts. These challenges can in part be addressed by gaining a better understanding of the tool. We believe that the three approaches of visibility, simplicity and practice are worth considering in future designs. Some point to radical redesign and require new technologies that go far beyond what today's beacons supply. Yet by deeply rethinking the way beacons shape interactions between rescuers on scene, we argue that better rescue and training tools could be created. Our future work will focus on furthering our understanding of collaborative avalanche rescue with beacons and on the development of concepts and prototypes for future beacons and training tools.

\section{ACKNOWLEDGMENTS}

We thank all the backcountry recreationists and the pro patrollers at Mount Baker who participated in the study.

\section{REFERENCES}

1. Adams, L. A systems approach to human factors and expert decision-making within Canadian avalanche phenomena. 2005.

2. Brugger, H. and Falk, M. Analysis of avalanche safety equipment for backcountry skiers. Avalanche News (66), (2004), 34-38.

3. Buxton, B. Sketching User Experiences: Getting the Design Right and the Right Design. Morgan Kaufmann, 2007.

4. Creswell, J.W. Qualitative Inquiry and Research Design: Choosing Among Five Approaches. SAGE, 2012.

5. Daffern, T. Backcountry Avalanche Safety: Skiers, Climbers, Boarders, Snowshoers. Rocky Mountain Books Ltd, 2009.

6. Edgerly, B. and Baugher, P. Talking the Talk: Human Factors, Group Communication, and the Next Frontier in Snow Safety. Proc, ISSW 2012,
7. Endsley, M.R. Toward a Theory of Situation Awareness in Dynamic Systems. Human Factors: The Journal of the Human Factors and Ergonomics Society 37, 1 (1995), 32-64.

8. Falk, M., Brugger, H., and Adler-Kastner, L. Avalanche survival chances. 368, 6466 (1994), 21-21.

9. Hereford, J. and Edgerly, B. $457 \mathrm{khz}$ electromagnetism and the future of avalanche transceivers. Proc. ISSW, (2000).

10. Hollan, J., Hutchins, E., and Kirsh, D. Distributed cognition: toward a new foundation for humancomputer interaction research. ACM Trans. Comput.Hum. Interact. 7, 2 (2000), 174-196.

11. Hutchins, E. Cognition in the Wild. Mit Press, 1995.

12. Jiang, X., Chen, N., Hong, J.I., Wang, K., Takayama, L., and Landay, J. Siren: Context-aware Computing for Firefighting. In A. Ferscha \& F. Mattern, eds., Pervasive Computing. Springer, 2004, 87-105.

13. MacMillan, J., Entin, E.E., and Serfaty, D. Communication overhead: The hidden cost of team cognition. Team cognition: Process and performance at the interand intra-individual level. American Psychological Association, Washington, DC, (2004).

14. McCammon, I. Heuristic Traps in Recreational Avalanche Accidents: Evidence and Implications. Avalanche News (68), (2004), 1-10.

15. Michahelles, F., Matter, P., Schmidt, A., and Schiele, B. Applying wearable sensors to avalanche rescue. Computers \& Graphics 27, 6 (2003), 839-847.

16. O'Bannon, A. Allen and Mike's Really Cool Backcountry Ski Book, Revised and Even Better!: Traveling and Camping Skills for a Winter Environment. Globe Pequot, 2007.

17. Radwin, M.I. and Grissom, C.K. Technological Advances in Avalanche Survival. Wilderness \& Environmental Medicine 13, 2 (2002), 143-152.

18.The American Institute for Avalanche Research and Education. The American Institute for Avalanche Research and Education. http://avtraining.org/.

19. Toups, Z. and Kerne, A. Implicit coordination in firefighting practice: design implications for teaching fire emergency responders. Proc. CHI'07, ACM, 707.

20. Yin, R.K. Case Study Research: Design and Methods. SAGE, 2009. 